\title{
Diagnostic and prognostic computed tomography imaging markers in basilar artery occlusion (Review)
}

\author{
RARES CRISTIAN FILEP ${ }^{1}$, LUCIAN MARGINEAN ${ }^{1}$, ADINA STOIAN $^{2}$ and ZOLTAN BAJKO ${ }^{3}$ \\ ${ }^{1} \mathrm{PhD}$ School of Medicine, and Departments of ${ }^{2}$ Pathophysiology and ${ }^{3}$ Neurology, 'George Emil Palade' \\ University of Medicine, Pharmacy, Sciences and Technology, 540142 Târgu Mureș, Romania
}

Received May 5, 2021; Accepted June 4, 2021

DOI: $10.3892 /$ etm.2021.10386

\begin{abstract}
Acute ischemic stroke treatment has been revolutionized by the addition of mechanical and aspiration thrombectomy. Randomized controlled trials have proven beyond doubt, the substantial clinical impact of endovascular interventions in anterior circulation territory strokes. Unfortunately, patients with vertebrobasilar ischemic stroke could not be included in these early trials due to inherent clinical, radiological, and prognostic particularities of posterior circulation ischemia; thus, indications for the treatment of posterior fossa strokes and basilar artery occlusion (BAO) are mainly based on retrospective studies and registries. BAO carries high morbidity and mortality, despite the new improvements in endovascular therapy. Identifying patients who will likely benefit from invasive treatment and have a good clinical outcome resides in discovering clinical, biological, or imaging markers, that have prognostic implications. Such imaging markers have been described, especially
\end{abstract}

Correspondence to: Dr Adina Stoian, Department of Pathophysiology, 'George Emil Palade' University of Medicine, Pharmacy, Sciences and Technology, 50 Gheorghe Marinescu Street, 540142 Târgu Mureș, Romania

E-mail: cretadina@yahoo.com

Abbreviations: AICA, anterior-inferior cerebellar artery; BAO, basilar artery occlusion; HDBA, hyperdense basilar artery; pc-ASPECTS, Posterior Circulation Alberta Stroke Program Early CT Score; PMI, Pons-Midbrain Index; pc-CTA, Posterior Circulation CT Angiography Score; pc-CS, Posterior Circulation Collateral Score; BATMAN, Basilar Artery On CT Prognostic Score; AIS, acute ischemic stroke; TIA, transient ischemic attack; LVO, large vessel occlusion; EIC, early ischemic changes; MCA, middle cerebral artery; PCA, posterior cerebral artery; PICA, posterior inferior cerebellar artery; PCom, posterior communicating artery; NCCT, non-contrast CT; CTA, computed tomography angiography; CTASI, computed tomography angiography source images; NIHSS, National Institute of Health Stroke Scale; mRS, modified Rankin Scale; SCA, superior cerebellar artery; tPA, tissue-type plasminogen activator

Key words: acute ischemic stroke, basilar artery occlusion, computed tomography, imaging markers, angiography in the last decade. Hyperdense Basilar Artery Sign (HDBA), Posterior Circulations-Alberta Stroke Program Early CT Score (pc-ASPECTS), Pons-Midbrain Index (PMI), Posterior Circulation Collateral Score (pc-CS), Posterior Circulation CT Angiography Score (pc-CTA), and Basilar Artery on CT Prognostic Score (BATMAN), are computed tomography (CT) markers with properties that can aid the diagnosis of BAO and can independently predict clinical outcome. This paper aims to present a comprehensive review of these imaging signs to have a thorough understanding of their diagnostic and prognostic attributes.

\section{Contents}

1. Introduction and aim

2. Literature research methods

3. Hyperdense Basilar Artery (HDBA) sign

4. Posterior Circulation Acute Stroke Prognosis Early CT

Score (pc-ASPECTS)

5. Pons-Midbrain Index (PMI)

6. Posterior Circulation CT Angiography (pc-CTA) Score

7. Posterior Circulation Collateral Score (pc-CS)

8. Basilar Artery on Computed Tomography Angiography (BATMAN) Prognostic Score

9. Current challenges and future directions

10. Conclusions

\section{Introduction and aim}

Basilar artery occlusion (BAO) accounts for approximately $1 \%$ of all cases of acute ischemic stroke (AIS) or transient ischemic attacks (TIA), and for 5\% of all intracranial large vessel occlusions (LVO) $(1,2)$. Although endovascular treatment is the gold-standard for anterior circulation LVO, its positive impact on the vertebrobasilar system is still a matter of debate (3). Hopefully, ongoing randomized trials will shed more light on this devastating pathology (4). Due to the misleading nature of prodromal symptoms related to $\mathrm{BAO}$, clinical diagnosis is often delayed, leading to prolonged time intervals to imaging evaluation and treatment (5).

Cross-sectional imaging has proven its diagnostic and prognostic utility for anterior circulation LVO shifting the 
paradigm from time is brain to imaging is brain (6). Computed tomography (CT) is the main imaging modality for the diagnosis of suspected AIS owing to its widespread availability in emergency departments. CT has a sensitivity of up to $80 \%$ for the detection of early ischemic changes (EIC) in middle cerebral artery (MCA) territory strokes (7). For posterior circulation ischemia, however, detection of EIC is difficult, mainly due to posterior fossa radiologic particularities, such as beam hardening artifacts, which limit the sensitivity to only $55 \%$ for cerebellar EIC, and $33 \%$ for brainstem EIC, respectively (8). Magnetic resonance imaging (MRI) is superior to $\mathrm{CT}$ in terms of sensitivity, especially in posterior fossa ischemia. However, the lack of available MRI machines in smaller hospitals or in developing countries, and limitations related to artifacts, uncooperative or severe patients, limit the feasibility of MRI evaluation in posterior ischemic strokes (9). Our aim is to review the imaging signs, or scores described on non-contrast CT (NCCT) and CT angiography (CTA) in acute $\mathrm{BAO}$ in order to provide an integrated view on their diagnostic and prognostic strengths and weaknesses.

\section{Literature research methods}

We performed a non-systematic PubMed search for papers between 1980 and 2019, using the terms 'basilar artery occlusion' and 'basilar artery thrombosis' and 'imaging'. Inclusion criteria were retrospective or prospective studies that evaluated diagnostic and prognostic computed-tomography changes in patients with BAO. The search generated 445 results. Furthermore, we identified papers related to imaging markers by searching the reference list of articles retrieved by our initial search. Only English reports were included. The final reference list is based on the relevance and originality of this review's objective and includes 14 total studies.

\section{Hyperdense Basilar Artery (HDBA) sign}

A hyperdense vessel is seen as a region of high attenuation on NCCT scans (Fig. 1). Pathologically it represents, in the appropriate clinical setting, an acute thrombus within the artery. The hyperdense appearance of fresh intravascular clots is determined by the extravasation of serum out of the clot, followed by a relative increase in concentration of red blood cells. Higher densities are mainly caused by the protein fraction of hemoglobin, much less by its iron content, which contributes only to $7-8 \%$ to its attenuation (10). Thrombi retrieved from patients with a dense MCA have, on average, a higher content of red blood cells (11-14) and are more frequently associated with a cardioembolic stroke subtype (15). These observations are not confirmed for BAO.

The HDBA sign can be a useful tool for the detection of BAO, with a sensitivity ranging between 68 and $94 \%$ and a specificity between 80 and $98 \%$ (16-18). Sensitivity is higher if the clinical diagnosis of BAO is highly probable (14) or if the attenuation of the hyperdense vessel is measured. Optimal measured cut-off values that best discriminate a HDBA, range between 40 and $46 \mathrm{HU}(17,18)$. Another method by which to increase detection of an HDBA, that was confirmed for dense MCAs, is to obtain a CT slice thickness of less than $2 \mathrm{~mm}$ or more appropriate $1 \mathrm{~mm}$, and to view them on 5 -mm-thick maximum intensity projection (MIP) reconstructions (19). One possible limitation of early studies evaluating the diagnostic value of the HDBA sign, is the use of a larger slice thickness, of $2 \mathrm{~mm}(16)$ or $4-5 \mathrm{~mm}(16,17)$, respectively. It is well known that a larger slice thickness increases partial volume averaging with cerebrospinal fluid and adjacent brain parenchyma, and can lead to false-negative NCCT results, potentially missing more hyperdense clots (18).

A dense basilar artery basilar artery (BA) sign is also valuable as a prognostic marker. It correlates well with discharge NIHSS and can independently predict short- (OR, 5.5; 95\% CI, 2.2-13.6; $\mathrm{P}<0.001)(20)$ and long-term outcome (OR, 5.3; 95\% CI, 1.1-33.3; P=0.05) (16). Nevertheless, in the absence of the HDBA sign, a brainstem ischemia or LVO should not be excluded; further contrast-enhanced vessel imaging is mandatory for the correct radiologic evaluation of patients with suspected posterior circulation stroke.

\section{Posterior Circulation Acute Stroke Prognosis Early CT Score (pc-ASPECTS)}

pc-ASPECTS is a 10-point score that evaluates EIC changes in the thalamus, occipital cortex, cerebellar hemispheres, midbrain, and pons. For the first three, 1 point is subtracted for each part if EIC are present, while 2 points each are subtracted if the midbrain or the pons are affected (21) (Fig. 2). Evaluation of the score is difficult on NCCT, due to posterior fossa beam hardening artefacts, particularly in the brainstem. CTA source images (CTASI) are a better tool for the visualization of ischemia. Parenchymal hypodensity on CTASI most likely represents a region of cerebral blood volume reduction (CBV). The extent of this region correlates well with admission and 24-h NIHSS, and with the 90-day functional outcome. Furthermore, ischemic lesion volume on CTASI does not differ significantly from DWI volume on MRI. If applied on CTASI, pc-ASPECTS is more sensitive and specific (65 and 82\%) (22). In their retrospective cohort of 46 patients with BAO, Puetz et al dichotomized the patients into two groups: CTASI pc-ASPECTS $\geq 8$ and $<8$. Patients with a score $\geq 8$ were 11 times more likely to have a good outcome (mRS $\leq 3): 52 \%$ achieved a good functional outcome at three months, whereas only $4 \%$ if the score was $<8$ (unadjusted RR, 12.1; 95\% CI, 1.7-84.9). Mortality was reduced by $60 \%$ if the score was $\geq 8$. If successful recanalization was obtained, $70 \%$ of the patients achieved a good prognosis if pc-ASPECTS was $\geq 8$, and only $9 \%$ if the score was $<8$ (unadjusted RR, 7.7; 95\% CI, 1.1-52.1) (21).

In patients from the Basilar Artery International Cooperation Study (BASICS) group, a significant association was found between pc-ASPECTS $\geq 8$, favorable outcome, functional independence $(\mathrm{mRS} \leq 2)$ and reduced mortality (23). However, after adjustment for age, NIHSS and tissue-type plasminogen activator (tPA), only functional independence (RR, 2.0; 95\% CI, 1.1-3.8) and mortality (RR, 0.7; 95\% CI, 0.5-0.98) were significantly associated, but not a favorable outcome. In a post hoc analysis, the same group was further dichotomized by pc-ASPECTS of $<6$ and $\geq 6$. It was found that a score of $\geq 6$ was an independent predictor of a favorable outcome, even after adjustment for age, NIHSS, and treatment modality (RR, 3.1; 95\% CI, 1.2-7.5) (23). In patients 
A

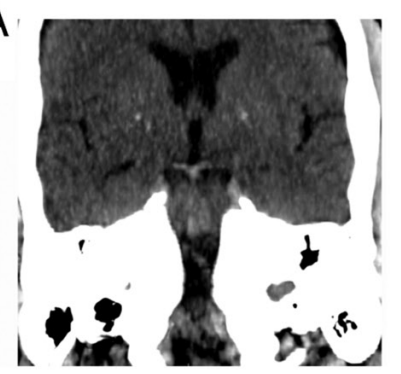

$\mathrm{B}_{\text {I }}$

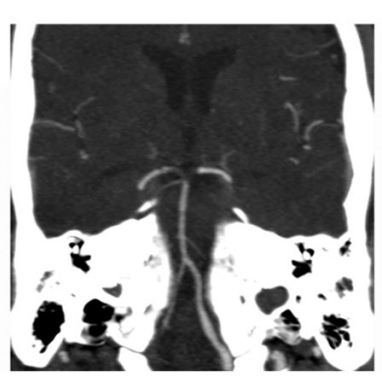

C

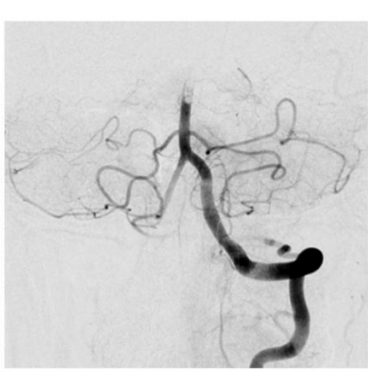

$\mathrm{D}$

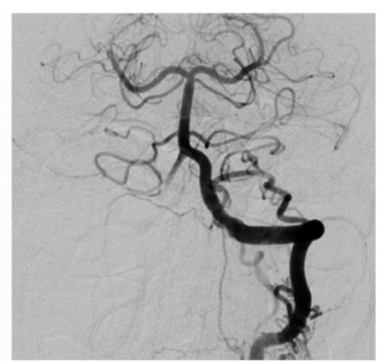

Figure 1. (A and B) Coronal reformated NCCT and CTA images reveal the HDBA sign and the corresponding distal BA thrombus, extending in both posterior cerebral arteries. (C and D) Before and after aspiration thrombectomy: Frontal projection digital subtraction angiography images confirm the BA occlusion, and subsequent recanalization. NCCT, non-contrast computer tomography; CTA, computer tomography angiography; HDBA, hyperdense basilar artery; BA, basilar artery.
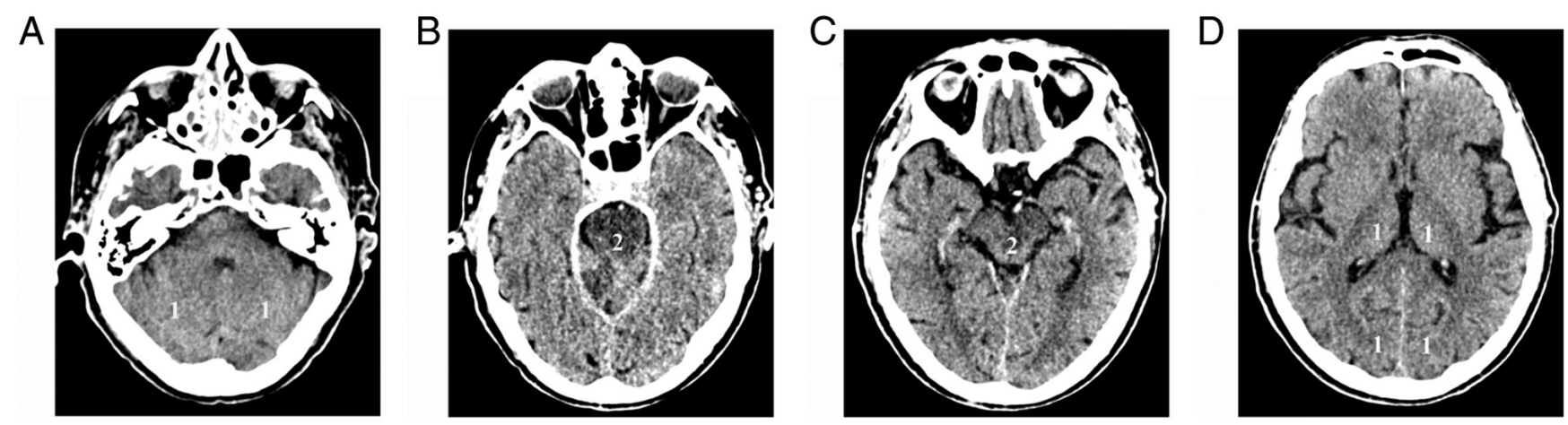

Figure 2. Axial non-contrast CT images (A) at the level of the cerebellar hemispheres, (B) pons, (C) midbrain and cerebral peduncles, and (D) thalamus, show the allocation of points to each structure for the calculation of pc-ASPECTS. CT, computed tomography; pc-ASPECTS, pc-ASPECTS, Posterior Circulation Alberta Stroke Program Early CT Score.

with BAO presenting with coma, the discriminative prognostic power of pc-ASPECTS of $<8 \mathrm{OR}, \geq 8$ was not found to be significantly associated with a favorable outcome or mortality, if adjusted for age, NIHSS and treatment modality (24). Even in a larger cohort comprising 231 patients with acute BAO, the prognostic value of pc-ASPECTS in predicting functional independence at 3 months was not significant (25).

Pc-ASPECTS dichotomized by $<8$ or $\geq 8$ was also evaluated on CT perfusion (CTP) maps in 27 patients from the BASICS registry (26). The most frequent changes were seen in $93 \%$ of cases on mean transit time (MTT) parameter maps (95\% CI, 76-99). Cerebral blood volume (CBV) pc-ASPECTS $<8$ was evident in 3 cases, all of whom died. However, none of the perfusion changes were associated with functional outcome, likely due to the small number of available cases (26).

\section{Pons-Midbrain Index (PMI)}

Schaefer et al more precisely attributed functional outcome to lesions located in the pons and midbrain, for which they developed the Pons-Midbrain Index, a scoring system based on CTASI: 0, if no ischemic changes are visible, 1 if $<50 \%$ of the territory is hypodense, 2 if $>50 \%$ of the parenchyma is hypodense. Brain structures scored were the medulla, pons, midbrain, thalami, occipital lobes, inferior parietal lobes and middle temporal lobes. Each structure was scored separately. The only regions that were correlated with death and disability were the pons and midbrain, which taken together as a sum of the scores, they called the PMI. A PMI $\geq 3$, or $<3$, was independently associated with mortality, or survival, respectively (27).

The Basilar Artery International Cooperation Study (BASICS) group looked at the death rate among patients with BAO who presented with coma. Among 78 patients with BAO and coma, $49 \%$ died, and $17 \%$ had a favorable outcome (mRS 0-3) if PMI was <3, as opposed to $76 \%$ deaths, and only $14 \%$ favorable outcome if PMI was $\geq 3$ (24). However, after adjusting for age, NIHSS and treatment type, only mortality was associated with a PMI <3 (RR, 0.67; 95\% CI, 0.46-1.00), but not a favorable outcome. Notably, a subgroup of patients, despite presenting with coma, with extensive pontine-mesencephalic ischemia $(\mathrm{PMI} \geq 3)$ had a favorable outcome at 1 month, comprising $14 \%$. Based on this observation, the authors argue against the use of the PMI to exclude patients with BAO and coma from intravenous or intraarterial treatment (24).

\section{Posterior Circulation CT Angiography (pc-CTA) score}

The Posterior Circulation CT Angiography (pc-CTA) score is a 6-point scoring system that evaluates the extent of the occlusion and, indirectly, the collateral circulation (28) (Fig. 3). One point is assigned for each of the following occluded segments: One of the vertebral arteries, proximal segment of the BA [from the vertebrobasilar junction to the origin of the anterior-interior cerebellar arteries (AICA)], middle segment of the 

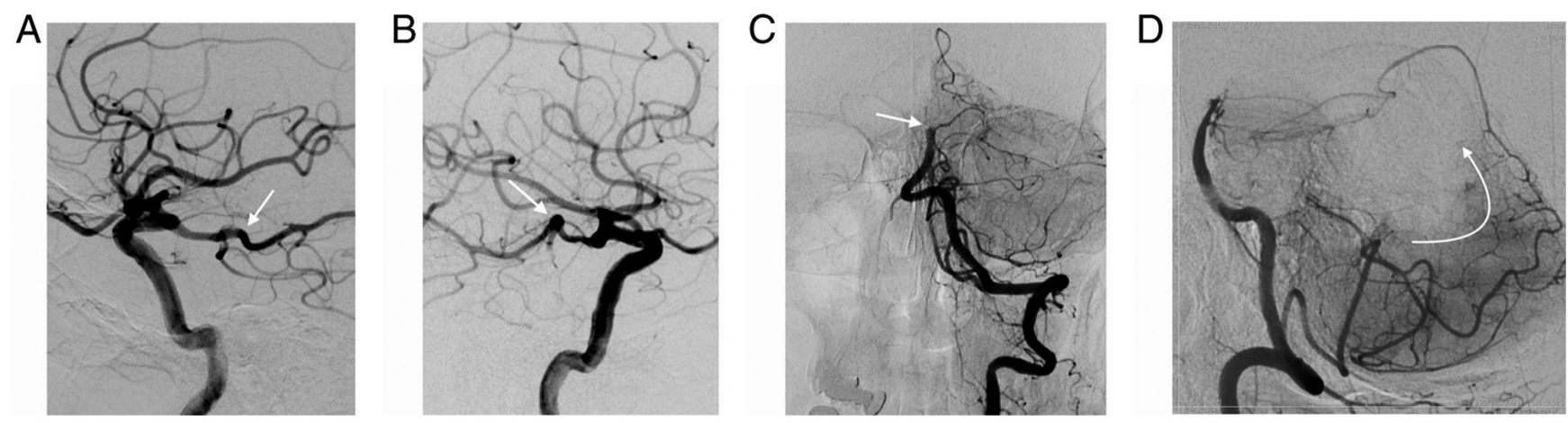

Figure 3. (A and B) Digital subtraction angiograms from bilateral internal carotid artery contrast injection show both posterior communicating arteries with a fetal configuration (white arrow) in a patient with basilar artery occlusion. (C and D) Contrast injection in the left vertebral artery reveal the distal basilar occlusion (white arrow) and collateral reconstitution of the superior cerebellar arteries through the posterior inferior cerebellar artery (curved arrow).

BA [from the origin of the AICA to the superior cerebellar arteries (SCA)], distal BA (from the SCA to the tip) and the posterior cerebral arteries (PCA). A pc-CTA score of 0 means that all vascular segments are open; a score of 6 , that all are occluded (28).

In their retrospective series of 15 patients, the primary endpoint was the correlation between the score and patient outcome at 3 months, as assessed by the modified Rankin Scale (mRS). A pc-CTA score $<3$ was associated with a good outcome ( $m R S \leq 3)$ in all patients. In contrast, the contrary, if the score was $\geq 3$, patient outcome was worse (28). In a retrospective study performed by Alemseged et al (29) a pc-CTA $\geq 3$ predicted a poor outcome if adjusted for age (OR, 1.6; 95\% CI, 1.1-2.1; P=0.008), but not for NIHSS.

\section{Posterior Circulation Collateral Score (pc-CS)}

The investigators enrolled in the Basilar Artery International Cooperation Study (BASICS) developed this CT angiography score [Posterior Circulation Collateral Score (pc-CS)] as a prognostic tool for identifying patients who present with a poor outcome (30). The score allots 1 point for each patent posterior inferior cerebellar artery (PICA), anterior-inferior cerebellar artery (AICA), superior cerebellar artery (SCA) and posterior communicating artery (Pcom) if its diameter is smaller than the ipsilateral PCA, or 2 points if it has a diameter equal to, or larger than the ipsilateral PCA. A score of 10 implies that all the aforementioned branches are patent, while a score of 0 , means that all of them are not visible/occluded. Three intervals of severity were defined for the pc-CS: Poor 0-3, intermediate 4-5, and good 6-10. Patients with a poor score had higher median NIHSS at admission and had more frequently severe symptoms (tetraplegia, locked-in state or coma), than patients with an intermediate or a good score. Moreover, there was a $25 \%$ lower risk of poor outcome in patients with a good pc-CS score, compared to those with a poor score, after adjustment for age, time to treatment and treatment modality (RR, 0.74; 95\% CI, 0.58-0.96). There was no significant difference between poor outcome compared to intermediate outcome. An important finding of their analysis was the crucial role played by the presence or absence of PComs, or their diameter. A poor outcome is more frequently encountered if one or both PComs are absent, and if their diameter is small (30).
Ravindren et al further emphasized the crucial importance of the PComs in BAO, by analyzing the presence or absence of collaterals, represented by the PCom and PICA-AICA anastomosis. Patients with collaterals had an almost 3-times higher likelihood of a good outcome at 3 months (OR, 2.73; 95\% CI, 1.01-7.39), while absence of both PComs was associated with a $60 \%$ decreased chance of good functional outcome at 90 days (OR, 0.39; 95\% CI, 0.17-0.93). Furthermore, even unilateral absence of a PCom led to a 2-fold increase in mortality (OR, 2.17; 95\% CI, 1.14-4.13) (25).

When time-to-treatment $\leq 6$ or $>6 \mathrm{~h}$ and revascularization status were taken into account, all patients with a favorable pc-CS had a good outcome (OR, 9.4; 95\% CI, 1.4-64; $\mathrm{P}=0.02)$. Conversely, an unfavorable pc-CS was associated with good outcome only in patients treated within $6 \mathrm{~h}$ from symptom onset, and not beyond (OR, 5.5; 95\% CI, 1.4-2; $\mathrm{P}=0.01)(31)$.

\section{Basilar Artery on Computed Tomography Angiography (BATMAN) Prognostic Score}

BATMAN is a 10-point score that incorporates thrombus burden and extent of primary collaterals. It allocates 1 point for the patency of either the vertebral artery, proximal BA segment, middle BA, distal BA, for each PCA and 2 points for each PCom larger than $1 \mathrm{~mm}$ in diameter. If smaller, i.e. hypoplastic, 1 point is given to each. If a fetal PCom is present, it receives 3 points (29). The optimal cut-off value to discriminate between good and bad functional outcome is a score of 7. A BATMAN score $<7$ is an independent predictor of poor outcome $(\mathrm{OR}, 6.9 ; 95 \% \mathrm{CI}, 1.4-33 ; \mathrm{P}=0.01)$ and an increased risk of mortality (OR, 7.4; 95\% CI, 1.2-44; $\mathrm{P}=0.03$ ) after adjustment for age and NIHSS. The score did not influence recanalization success between the two groups. If recanalization was not achieved, the rates of poor outcome were similar. However, if recanalization was successful, only $24 \%$ of the patients had a poor outcome if BATMAN was $\geq 7$, compared to $76 \%$ if $<7$. Compared to the prognostic scores reviewed earlier, BATMAN performed better than pc-CS in terms of accuracy and interrater agreement, but not better than pc-CTA (29).

In a recent paper, Alemseged et al (31) assessed the prognostic value of BATMAN correlated with 
time-to-treatment (TTT) and recanalization status (mTICI). In patients with a favorable score treated within or even beyond $6 \mathrm{~h}$, complete revascularization (mTICI 2b-3) was associated with a good outcome (adjusted OR, 15.8; 95\% CI, 1.4-175; $\mathrm{P}=0.02$ ). However, in cases with an unfavorable score, revascularization was associated with good outcome only if obtained within $6 \mathrm{~h}(\mathrm{OR}, 15 ; 95 \% \mathrm{CI}, 1.9-124 ; \mathrm{P}=0.01)$; findings that support the relevance of collaterals and thrombus load in late time-windows for BAO.

\section{Current challenges and future directions}

Major limitations of the aforementioned studies are related to their retrospective design and relatively small number of patients (see Tables SI and SII). Furthermore, the heterogenous treatment modalities (intravenous tPA, intraarterial thrombolysis, mechanical thrombectomy) and different clinical and imaging outcome parameters reported ( $\mathrm{mRS} \leq 2$ or $\leq 3$, mTICI), limit the generalizability of the scores, especially in the new era of mechanical and aspiration thrombectomy. There are also restrictions imposed by spiral computed tomography physics; posterior fossa beam hardening artefacts decrease sensitivity of diagnostic tests, particularly for the visualization of parenchymal structures.

Therefore, prospective trials, using standardized computed tomographic parameters, clinical outcome and recanalization scales are mandatory in order to consolidate the diagnostic and prognostic value of these imaging markers; emphasis should be placed on collateral capacity, thrombus burden and thrombus location. Moreover, occlusion etiology should be dichotomized into atherosclerotic vs. cardioembolic, in light of their different treatment strategies and prognosis (32). There is a growing body of evidence that supports, in addition to the classic non-contrast CT and CT angiography, the inclusion of perfusion imaging in the armamentarium aimed at the patient with posterior fossa ischemia (26,33-35). It improves detection of infratentorial ischemia and provides further prognostic information. Ultimately, machine learning software could be used to integrate the different imaging parameters and to guide treatment decision-making.

\section{Conclusions}

Acute BAO remains a devastating disease in spite of recent technological improvements. Computed tomography markers can aid and accelerate the diagnosis of this complex clinical entity, leading to shorter time-to-treatment intervals. They can provide valuable prognostic knowledge to better inform patients and their relatives about the consequences of the disease. However, none of the computed tomography markers should be used to exclude patients from intravenous and intraarterial therapy.

\section{Acknowledgements}

Not applicable.

\section{Funding}

No funding was received.

\section{Availability of data and materials}

All information included in this review is documented by relevant references.

\section{Authors' contributions}

RCF conceived the concept of the review; LM, AS, and ZB performed the literature search, analyzed the relevant literature and wrote the manuscript. RCF, ZB, and AS contributed to the interpretation of the data and the revision of the manuscript, and provided critical review for the manuscript. All authors read and approved the final manuscript for publication.

\section{Ethics approval and consent to participate}

Not applicable.

\section{Patient consent for publication}

Not applicable.

\section{Competing interests}

The authors declare that they have no competing interests.

\section{Authors' information}

Rares Cristian Filep: ORCID ID: 0000-0002-7422-1178.

\section{References}

1. Mattle HP, Arnold M,Lindsberg PJ, Schonewille WJ and Schroth G: Basilar artery occlusion. Lancet Neurol 10: 1002-1014, 2011.

2. Kayan Y, Meyers PM, Prestigiacomo CJ, Kan P and Fraser JF: Society of NeuroInterventional Surgery: Current endovascular strategies for posterior circulation large vessel occlusion stroke: Report of the Society of NeuroInterventional Surgery Standards and Guidelines Committee. J Neurointerv Surg 11: 1055-1062, 2019.

3. Turc G, Bhogal P, Fischer U, Khatri P, Lobotesis K, Mazighi M, Schellinger PD, Toni D, de Vries J, White P and Fiehler J: European stroke organisation (ESO) - European society for minimally invasive neurological therapy (ESMINT) guidelines on mechanical thrombectomy in acute ischemic stroke. J Neurointerv Surg 11: 535-538, 2019.

4. van der Hoeven EJRJ, Schonewille WJ, Vos JA, Algra A, Audebert HJ, Berge E, Ciccone A, Mazighi M, Michel P, Muir KW, et al: The basilar artery international cooperation study (BASICS): Study protocol for a randomised controlled trial. Trials 14: 200, 2013.

5. Sarraj A, Medrek S, Albright K, Martin-Schild S, Bibars W, Vahidy F, Grotta JC and Savitz SI: Posterior circulation stroke is associated with prolonged door-to-needle time. Int J Stroke 10: 672-678, 2015.

6. Puig J, Shankar J, Liebeskind D, Terceño M, Nael K, Demchuk AM, Menon B, Dowlatshahi D, Leiva-Salinas C, Wintermark M, et al: From 'time is brain' to 'imaging is brain': A paradigm shift in the management of acute ischemic stroke. J Neuroimaging 30: 562-571, 2020.

7. Grotta JC, Chiu D, Lu M, Patel S, Levine SR, Tilley BC, Brott TG, Haley EC Jr, Lyden PD, Kothari R, et al: Agreement and variability in the interpretation of early CT changes in stroke patients qualifying for intravenous rtPA therapy. Stroke 30: 1528-1533, 1999.

8. Hwang DY, Silva GS, Furie KL and Greer DM: Comparative sensitivity of computed tomography vs. magnetic resonance imaging for detecting acute posterior fossa infarct. J Emerg Med 42: 559-565, 2012. 
9. Schramm P, Schellinger PD, Klotz E, Kallenberg K, Fiebach JB, Külkens S, Heiland S, Knauth M and Sartor K: Comparison of perfusion computed tomography and computed tomography angiography source images with perfusion-weighted imaging and diffusion-weighted imaging in patients with acute stroke of less than 6 h' duration. Stroke 35: 1652-1658, 2004.

10. New PF and Aronow S: Attenuation measurements of whole blood and blood fractions in computed tomography. Radiology 121 (3 Pt. 1): 635-640, 1976.

11. Liebeskind DS, Sanossian N, Yong WH, Starkman S, Tsang MP, Moya AL, Zheng DD, Abolian AM, Kim D, Ali LK, et al: CT and MRI early vessel signs reflect clot composition in acute stroke. Stroke 42: 1237-1243,2011.

12. Bajkó Z, Maier S, Moţăţăianu A, Balasa R, Vasiu S, Stoian A and Andone S: Stroke secondary to traumatic carotid artery injury A case report. J Crit Care Med (Targu Mures) 4: 23-28, 2018.

13. Bajkó Z, Bălaşa R, Moţăţăianu A, Bărcuţean L, Stoian A, Stirbu N and Maier S: Malignant middle cerebral artery infarction secondary to traumatic bilateral internal carotid artery dissection. A case report. J Crit Care Med (Targu Mures) 2: 135-141, 2016.

14. Filep RC, Bajko Z, Simu IP and Stoian A: Pseudo-dissection of the internal carotid artery in acute ischemic stroke. Acta Neurol Belg 120: 469-472, 2020.

15. Kim SK, Baek BH, Lee YY and Yoon W: Clinical implications of CT hyperdense artery sign in patients with acute middle cerebral artery occlusion in the era of modern mechanical thrombectomy. J Neurol 264: 2450-2456, 2017.

16. Goldmakher GV, Camargo EC, Furie KL, Singhal AB, Roccatagliata L, Halpern EF, Chou MJ, Biagini T, Smith WS, Harris GJ, et al: Hyperdense basilar artery sign on unenhanced $\mathrm{CT}$ predicts thrombus and outcome in acute posterior circulation stroke. Stroke 40: 134-139, 2009.

17. Connell L, Koerte IK, Laubender RP, Morhard D, Linn J, Becker HC, Reiser M, Brueckmann H and Ertl-Wagner B: Hyperdense basilar artery sign-a reliable sign of basilar artery occlusion. Neuroradiology 54: 321-327, 2011.

18. Ernst M, Romero JM, Buhk JH, Cheng B, Herrmann J, Fiehler J and Groth M: Sensitivity of hyperdense basilar artery sign on non-enhanced computed tomography. PLoS One 10: e0141096, 2015.

19. Riedel CH, Zoubie J, Ulmer S, Gierthmuehlen J and Jansen O: Thin-slice reconstructions of nonenhanced CT images allow for detection of thrombus in acute stroke. Stroke 43: 2319-2323, 2012.

20. Tan X, and Guo Y: Hyperdense basilar artery sign diagnoses acute posterior circulation stroke and predicts short-term outcome. Neuroradiology 52: 1071-1078, 2010.

21. Puetz V, Sylaja PN, Coutts SB, Hill MD, Dzialowski I, Mueller P, Becker U, Urban G, O'Reilly C, Barber PA, et al: Extent of hypoattenuation on CT angiography source images predicts functional outcome in patients with basilar artery occlusion. Stroke 39: 2485-2490, 2008

22. Bhatia R, Bal SS, Shobha N, Menon BK, Tymchuk S, Puetz V, Dzialowski I, Coutts SB, Goyal M, Barber PA, et al: CT angiographic source images predict outcome and final infarct volume better than noncontrast CT in proximal vascular occlusions. Stroke 42: 1575-1580, 2011

23. Puetz V, Khomenko A, Hill MD, Dzialowski I, Michel P, Weimar C, Wijman CA, Mattle HP, Engelter ST, Muir KW, et al: Extent of hypoattenuation on CT angiography source images in basilar artery occlusion: Prognostic value in the Basilar Artery International Cooperation Study. Stroke 42: 3454-3459, 2011

24. Pallesen LP, Khomenko A, Dzialowski I, Barlinn J, Barlinn K, Zerna C, van der Hoeven EJ, Algra A, Kapelle LJ, Michel P, et al: CT-angiography source images indicate less fatal outcome despite coma of patients in the Basilar Artery International
Cooperation Study. Int J Stroke 12: 145-151, 2017.

25. Ravindren J, Aguilar Pérez M, Hellstern V, Bhogal P, Bäzner H and Henkes H: Predictors of outcome after endovascular thrombectomy in acute basilar artery occlusion and the $6 \mathrm{hr}$ time window to recanalization. Front Neurol 10: 923, 2019.

26. Pallesen LP, Gerber J, Dzialowski I, van der Hoeven EJ, Michel P, Pfefferkorn T, Ozdoba C, Kappelle LJ, Wiedemann B, Khomenko A, et al: Diagnostic and prognostic impact of pc-ASPECTS applied to perfusion CT in the Basilar Artery International Cooperation Study. J Neuroimaging 25: 384-389, 2015.

27. Schaefer PW, Yoo AJ, Bell D, Barak ER, Romero JM, Nogueira RG, Lev MH, Schwamm LH, Gonzalez RG and Hirsch JA: CT Angiography-Source image hypoattenuation predicts clinical outcome in posterior circulation strokes treated with Intra-Arterial therapy. Stroke 39: 3107-3109, 2008.

28. Da Ros V, Meschini A, Gandini R, Del Giudice C, Garaci F, Stanzione P, Rizzato B, Diomedi M, Simonetti G, Floris R and Sallustio F: Proposal for a vascular computed Tomography-Based grading system in posterior circulation stroke: A Single-Center experience. J Stroke Cerebrovasc Dis 25: 368-377, 2016.

29. Alemseged F, Shah DG, Diomedi M, Sallustio F, Bivard A, Sharma G, Mitchell PJ, Dowling RJ, Bush S, Yan B, et al: The basilar artery on computed tomography angiography prognostic score for basilar artery occlusion. Stroke 48: 631-637, 2017.

30. van der Hoeven EJ, McVerry F, Vos JA, Algra A, Puetz V, Kappelle LJ and Schonewille WJ; BASICS registry investigators: Collateral flow predicts outcome after basilar artery occlusion: The posterior circulation collateral score. Int J Stroke 11: 768-775, 2016.

31. Alemseged F, Van der Hoeven E, Di Giuliano F, Shah D, Sallustio F, Arba F, Kleinig TJ, Bush S, Dowling RJ, Yan B, et al: Response to Late-Window endovascular revascularization is associated with collateral status in basilar artery occlusion. Stroke 50: 1415-1422, 2019.

32. Piechowiak EI, Kaesmacher J, Zibold F, Dobrocky T, Mosimann PJ, Jung S, Fischer U, Arnold M, Bellwald S, Heldner MR, et al: Endovascular treatment of tandem occlusions in vertebrobasilar stroke: Technical aspects and outcome compared with isolated basilar artery occlusion. J Neurointery Surg 12: 25-29, 2020.

33. Bollwein C, Plate A, Sommer WH, Thierfelder KM, Janssen H, Reiser MF, Straube A and von Baumgarten L: Diagnostic accuracy of whole-brain CT perfusion in the detection of acute infratentorial infarctions. Neuroradiology 58: 1077-1085, 2016.

34. Fabritius MP, Reidler P, Froelich MF, Rotkopf LT, Liebig T, Kellert L, Feil K, Tiedt S, Kazmierczak PM, Thierfelder KM, et al: Incremental value of computed tomography perfusion for final infarct prediction in acute ischemic cerebellar stroke. J Am Heart Assoc 8: e013069, 2019.

35. Sporns P, Schmidt R, Minnerup J, Dziewas R, Kemmling A, Dittrich R, Zoubi T, Heermann P, Cnyrim C, Schwindt W, et al: Computed tomography perfusion improves diagnostic accuracy in acute posterior circulation stroke. Cerebrovasc Dis 41: 242-247, 2016.

This work is licensed under a Creative Commons Attribution-NonCommercial-NoDerivatives 4.0 International (CC BY-NC-ND 4.0) License. 